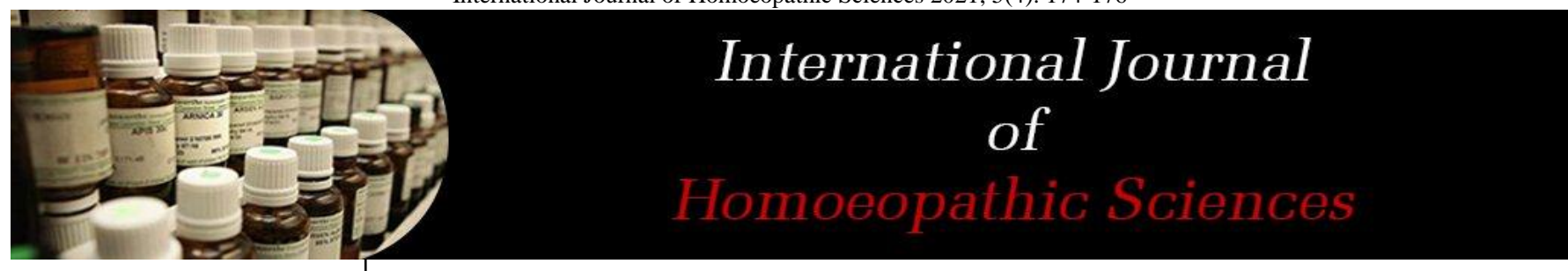

E-ISSN: 2616-4493 P-ISSN: 2616-4485 www.homoeopathicjournal.com IJHS 2021; 5(4): 174-176 Received: 26-07-2021 Accepted: 10-09-2021

Dr. Nandini Dadhich MD (PGR), Department of Materia Medica, Homoeopathy University, Jaipur, Rajasthan, India

Dr. Vanija Sharma Ph.D, Associate Professor Department of Materia Medica, Homoeopathy University, Jaipur, Rajasthan, India
Corresponding Author: Dr. Nandini Dadhich MD (PGR), Department of Materia Medica, Homoeopathy University, Jaipur, Rajasthan, India

\section{A guide to deal whooping cough in homoeopathy}

\author{
Dr. Nandini Dadhich and Dr. Vanija Sharma
}

DOI: $\underline{\text { https://doi.org/10.33545/26164485.2021.v5.i4c.468 }}$

\section{Abstract}

Whooping cough, also known as pertussis or the 100-day cough, is a highly contagious bacterial disease. Initial symptoms are usually similar to those of the common cold with a runny nose, fever, and mild cough, but these are followed by weeks of severe coughing fits. Following a fit of coughing, a high-pitched whoop sound or gasp may occur as the person breathes in. Prevention is mainly by vaccination with the pertussis vaccine. Antibiotics may be used to prevent the disease in those who have been exposed and are at risk of severe disease.

Keywords: Whooping cough, pertussis, homoeopathy, homoeopathic management

\section{Introduction}

Pertussis is an acute infection of the respiratory tract caused by Bordetella pertussis. The name pertussis means "violent cough," which aptly describes the most consistent and prominent feature of the illness. The inspiratory sound made at the end of an episode of paroxysmal coughing gives rise to the common name for the illness, "whooping cough." The Chinese name for pertussis is "the 100-day cough," which accurately describes the clinical course of the illness. Pertussis is a highly communicable disease, with attack rates of 80 $100 \%$ among unimmunized household contacts and $20 \%$ within households in wellimmunized populations. The infection has a worldwide distribution, with cyclical outbreaks every $3-5$ years ${ }^{[1,2]}$.

Pathogenesis: Infection with B. pertussis is initiated by attachment of the organism to the ciliated epithelial cells of the nasopharynx. At the site of attachment, the organism multiplies, producing a variety of other toxins that cause local mucosal damage. Systemic manifestations (lymphocytosis) result from the effects of the toxins. Detractors counter that pertussis toxin is not the critical factor because paroxysmal cough also occurs in patients infected with B. parapertussis, which does not produce pertussis toxin. It is thought that neurologic events in pertussis, such as seizures and encephalopathy, are due to hypoxia from coughing paroxysms or apnea rather than to the effects of specific bacterial products. B. pertussis pneumonia, which occurs in up to $10 \%$ of infants with pertussis, is usually a diffuse bilateral primary infection. In older children and adults with pertussis, pneumonia is often due to secondary bacterial infection with streptococci or staphylococci. Deaths from pertussis among young infants are frequently associated with very high levels of leukocytosis and pulmonary hypertension ${ }^{[1,2]}$.

Clinical Manifestations ${ }^{[1,2]}$ : After an incubation period of 1 to 3 weeks, pertussis infection typically progresses through three distinct stages:

- The catarrhal phase presents similarly to other upper respiratory tract infections, with fever, fatigue, rhinorrhea, and conjunctival injection. The catarrhal phase lasts 1 to 2 weeks and is the most infectious stage of the disease.

- The paroxysmal phase follows the catarrhal phase and is characterized by paroxysms of a staccato cough and the resolution of fever. The patient typically coughs repeatedly, followed by forceful inspiration which creates the characteristic "whoop." These episodes may be triggered by cold or noise, and are more common at night. Patients are nontoxic-appearing in between paroxysms, but during coughing episodes may exhibit cyanosis, diaphoresis, or apnea. Immediately following a paroxysm, patients may develop post-tussive emesis, syncope, or apnea.

- During the convalescent phase, a residual cough persists for weeks to months, usually triggered by exposure to another upper respiratory infection or irritant. 
Atypical presentations are common in infants, and fever may not occur. Rather, tachypnea, apnea, cyanosis, and episodic bradycardia may be the presenting features.

Increased intrathoracic pressure from coughing may result in petechiae above the nipple line, subconjunctival hemorrhage, and epistaxis.

Breath sounds are variable; auscultation may reveal clear lungs or rhonchi, while rales suggest superimposed pneumonia. The inspiratory whoop or gasp is usually heard in children between 6 months to 5 years.

\section{Diagnosis $[1,2[$}

- Nasopharyngeal culture and polymerase chain reaction (PCR) may yield laboratory confirmation.

- In adults, by the time the diagnosis is suspected, cultures are typically negative (96\%), and overall culture sensitivity is only $20 \%$ to $40 \%$. PCR is more sensitive and specific than culture.

- In the emergency department, pertussis should be considered in patients with prolonged cough, especially occurring in paroxysms or with whoops, or post-tussive emesis.

- During the late catarrhal and early paroxysmal phases, leukocytosis with lymphocytosis may raise suspicion for pertussis

- Chest x-ray findings are nonspecific and may show peribronchial thickening, atelectasis, or infiltrates. The classic association, though not often seen, is a "shaggy" right heart border.

\section{Differential diagnosis ${ }^{[1,2]}$}

- Pertussis initially presents similarly to other respiratory infections, such as viral upper respiratory infection, bronchiolitis, pneumonia, and tuberculosis.

- Foreign body aspiration should be considered in younger patients, and exacerbation of chronic obstructive pulmonary disease should be considered in older patients with the appropriate history.

- The striking leukocytosis may also be confused for leukemia.

\section{Complications ${ }^{[1,2]}$}

- Secondary pneumonia or otitis media may occur. The most common causes of secondary bacterial pneumonia are Streptococcus pneumoniae, Streptococcus pyogenes, Haemophilus influenzae, and Staphylococcus aureus; although viral infections with the respiratory syncytial virus, cytomegalovirus, and adenovirus superinfections are also common.

- CNS complications such as seizures and encephalopathy can occur, likely secondary to hypoxia, hypoglycemia, toxins, secondary infections, or cerebral bleeding from increased pressure during coughing.

- Sudden increases in intrathoracic and intraabdominal pressures can also result in periorbital edema, pneumothorax, pneumomediastinum, subcutaneous emphysema, diaphragmatic rupture, umbilical and inguinal hernias, and rectal prolapse.

- Pertussis toxin also causes histamine hypersensitivity and increased insulin secretion.

- Infants are particularly prone to bradycardia, hypotension, and cardiac arrest from pertussis.

\section{Treatment/management ${ }^{[1,2]}$}

- Treatment of pertussis is largely supportive, including oxygen, suctioning, hydration, and avoidance of respiratory irritants.

- Parenteral nutrition may be necessary as the disease tends to have a prolonged course.

- Hospitalization is indicated for patients with superimposed pneumonia, hypoxia, central nervous system (CNS) complications, or who are unable to tolerate nutrition and hydration by mouth.

- Neonates should be admitted to an intensive care setting as life-threatening cardiopulmonary complications and arrest can occur unexpectedly.

- Antibiotic effect on the duration or severity of the disease is minimal when started in the catarrhal phase, and not proven effective when started in the paroxysmal phase.

- Strict isolation is important while the patient remains infectious.

- Vaccination is recommended with the acellular vaccine at ages 2, 4, 6, 15-18 months, and at age 4-6 years.

\section{Homoeopathic treatment ${ }^{[3,4]}$}

- Aconitum Napellus: There is constant pressure in left chest; oppressed breathing on least motion. Hoarse, dry, croupy cough; loud, labored breathing. Child grasps at throat every time he coughs. Very sensitive to inspired air. Shortness of breath. Larynx sensitive. Stitches through chest. Cough, dry, short, hacking; worse at night and after midnight. Hot feeling in lungs. Blood comes up with hawking. Tingling in chest after cough.

- Antimonium Tartaricum: Hoarseness, great rattling of mucus, but very little is expectorated. Burning sensation in chest, which ascends to throat. Rapid, short, difficult breathing; seems as if he would suffocate; must sit up. Coughing and gaping consecutively. Cough excited by eating, with pain in chest and larynx. Dyspnoea relieved by eructation. Cough and dyspnoea better lying on right side.

- Arnica Montana: Coughs depending on cardiac lesion, paroxysmal, at night, during sleep, worse exercise. Cough produced by weeping and lamenting. Dry, from tickling low down in trachea. Bloody expectoration. Dyspnoea with haemoptysis. Violent spasmodic cough, with facial herpes. Whooping cough, child cries before coughing.

- Belladonna: Drying in nose, fauces, larynx, and trachea. Tickling, short, dry cough; worse at night. Larynx feels sore. Respiration oppressed, quick, unequal. Painless hoarseness. Cough with pain in left hip. Barking cough, whooping cough, with pain in stomach before attack, with expectoration of blood. Stitches in chest when coughing. Larynx very painful; feels as if a foreign body were in it, with cough.

- Castanea Vesca: A useful remedy in whooping-cough, especially in the early stage, with dry, ringing, violent, spasmodic cough. Desire for warm drinks.

- Coccus Cacti: Constant hawking from enlarged uvula; coryza, with inflamed fauces; accumulation of thick viscid mucus, which is expectorated with great difficulty. Tickling in larynx. Sensation of a crumb behind larynx, must swallow continually; brushing 
teeth causes cough. Fauces very sensitive. Suffocative cough; worse, first waking, with tough, white mucus, which strangles. Spasmodic morning cough. Whooping cough attacks end with vomiting of this tough mucus.

- Corallium Rubrum: Hawking of profuse mucus. Throat very sensitive, especially to air. Profuse, nasal catarrh. Inspired air feels cold. Profuse secretion of mucus dropping through posterior nares. Dry, spasmodic, suffocative cough; very rapid cough, short, barking. Cough with great sensitiveness of air-passages; feel cold on deep inspiration. Continuous hysterical cough. Feels suffocated and greatly exhausted after whooping-cough.

- Drosera Rotundifolia: Spasmodic, dry irritative cough, like whooping-cough, the paroxysms following each other very rapidly; can scarcely breathe; chokes. Cough very deep and hoarse; worse, after midnight; yellow expectoration, with bleeding from nose and mouth; retching. Deep, hoarse voice; hoarseness; laryngitis. Rough, scraping sensation deep in the fauces and soft palate. Laryngeal phthisis, with rapid emaciation.

- Hydrocyanicum Acidum: Dry, spasmodic, suffocative cough. Contraction of throat. Whooping-cough.

- Hyoscyamus Niger: Suffocating fits. Spasm, forcing bending forward. Dry, spasmodic cough at night (worse lying down; better sitting up), from itching in the throat, as if uvula were too long. Haemoptysis.

- Ipecacuanha: Dyspnoea; constant constriction in chest. Yearly attacks of difficult shortness of breathing. Cough incessant and violent, with every breath. Chest seems full of phlegm, but does not yield to coughing. Suffocative cough; child becomes stiff, and blue in the face. Whooping-cough, with nosebleed, and from mouth. Haemoptysis from slightest exertion. Hoarseness, especially at end of a cold.

- Justicia Adhatoda: Dry cough from sternal region all over chest. Hoarseness, larynx painful. Paroxysmal cough, with suffocative obstruction of respiration. Severe dyspnoea with cough. Tightness across chest. Asthmatic attacks, cannot endure a close, warm room. Whooping-cough.

- Kalium Muriaticum: Cough short, acute, and spasmodic, like whooping-cough; expectoration thick and white. Rattling sounds of air passing through thick, tenacious mucus in the bronchi; difficult to cough up.

- Mephitis: False croup; cannot exhale. Spasmodic and whooping-cough. Few paroxysms in day-time, but many at night; with vomiting after eating. Asthma, as if inhaling sulphur; cough from talking; hollow, deep, with rawness, hoarseness, and pains through chest. Violent spasmodic cough; worse at night.

- Sepia Officinalis: Dry, fatiguing cough, apparently coming from stomach. Oppression of chest morning and evening. Dyspnoea; worse, after sleep; better, rapid motion. Cough in morning, with profuse expectoration, tasting salty. Whooping-cough that drags on. Cough excited by tickling in larynx or chest.

- Trifolium Pratense: Hoarse and choking; chills with cough at night. Cough on coming into the open air. Spasmodic cough; whooping cough, paroxysms; worse at night.

\section{Conclusion}

In homoeopathy, medicines are prescribed on the basis of totality of symptoms but in many cases there occurs paucity of symptoms with some presentation of peculiar modality where certain medicines play significant role in the treatment. These medicines are well proved and have been found effective in clinical practice.

\section{References}

1. Lauria AM. Pertussis [Internet]. StatPearls [Internet]. U.S. National Library of Medicine; 2021 [cited 2021Sep10]. Available from: https://www.ncbi.nlm.nih.gov/books/NBK519008/

2. Jameson JL. Harrison's principles of Internal Medicine. New York, NY: McGraw-Hill Education 2018.

3. Boericke W. Boericke's New Manual of Homoeopathic Materia Medica with Repertory. Third revised and augmented edition based on $9^{\text {th }}$ edition. Noida, India: B. Jain Publishers (P) Ltd 2018.

4. Murphy Robin. Lotus Materia Medica. \#3 ${ }^{\text {rd }}$ edition. Noida, India: B. Jain Publishers (P) Ltd 2006. 